\title{
Wavelet Analysis of Cytological Preparations Image in Different Color Systems
}

\author{
Vyacheslav V. Lyashenko', Asaad MA. Babker2, Valentin A. Lyubchenko \\ ${ }^{1}$ Department of Informatics, Kharkiv National University of Radio Electronics, Kharkiv, Ukraine \\ ${ }^{2}$ Department of Medical Laboratories Science, College of Allied Health Science, Gulf Medical University, Ajman, UAE \\ Email: *azad.88@hotmail.com
}

How to cite this paper: Lyashenko, V.V., MA. Babker, A. and Lyubchenko, V.A. (2017) Wavelet Analysis of Cytological Preparations Image in Different Color Systems. Open Access Library Journal, 4: e3760. https://doi.org/10.4236/oalib.1103760

Received: June 23, 2017

Accepted: July 11, 2017

Published: July 14, 2017

Copyright $\odot 2017$ by authors and Open Access Library Inc.

This work is licensed under the Creative Commons Attribution International License (CC BY 4.0).

http://creativecommons.org/licenses/by/4.0/

\begin{abstract}
Processing of microscope images in medicine is one of the priority research areas. At the same time, the processing of images of cytological preparations occupies a special place. This is due to the fact that such studies allow for a comprehensive diagnosis of the state of human health, identify and prevent the development of diseases in the early stages. But this requires the results of processing the original images, which provide additional information. To do this, we investigate the possibility of using wavelet analysis in color models RGB and HSV. We showed the importance of using the HSV model for more information. It is shown that the procedure for changing the contrast in the HSV model is better than the contrast change procedure in the RGB model when using wavelet analysis.
\end{abstract}

\section{Subject Areas}

Histology

\section{Keywords}

Wavelet Analysis, Image, Contrast Enhancement, Cell, Medicine, Cytology Preparation, Color Systems

\section{Introduction}

Processing of different images is an important area in the study of the real world. Such an analysis makes it possible to obtain additional information. Additional information allows making correct and important decision. This is due to the fact that visual images are informative, but this information must be obtained. Currently, there are many systems that allow you to record and analyze the various images [1] [2] [3].

Image cytological preparation is an example of real-world objects. Analysis of 
cytological preparations image allows studying cell structure, determining the change in cell structure to count the various elements of the cell. This is the basis for a more in depth study of the human body, to study the dynamics of its operation and to diagnose possible diseases in the early stages of their development. Application of image processing techniques for analyzing cytological preparations imaging involves the solution of a complex of problems. This in turn involves the use of various image processing methods.

For example, S. Singh and R. Gupta [4], G. Mahendran, R. Babu and D. Sivakumar [5], examine the possibility of applying the texture analysis methods for cytology preparations. M. Saha, S. Agarwal, I. Arun, R. Ahmed, S. Chatterjee, P. Mitra, and C. Chakraborty, study the issues of threshold selection for cytological image segmentation [6]. Y. M. George, B. M. Bagoury, H. H. Zayed, and M. I. Roushdy, suggest conducting automated segmentation of cells in cytological image under study [7].

Nevertheless, the complexity of processing images of cytological preparations requires a constant study of methods for their analysis. One aspect of this processing is discussed in this article.

\section{Materials and Methods}

\subsection{Wavelets as a Tool for Analyzing Images of Cytological Preparations}

Among the methods of processing images of cytological preparations, we can distinguish:

-methods pre-processing of images. These methods allow improving the quality of image processing (noise removal, contrast enhancement);

-methods of image analysis. These methods allow you to obtain additional information (image segmentation, contour detection, wavelet analysis).

However, it should be taken into account that an integrated approach to image processing of cytological preparations should be applied. This approach assumes a close relationship between image pre-processing methods and image analysis methods. At the same time, this problem is very relevant in the processing of color images. This is due to the features of the representation and processing of color images. Therefore, it is important to consider together and the methods of pre-processing images and methods of image analysis.

Among the methods of analyzing the images of cytological preparations, one should single out the ideology of wavelet analysis. This is due to the fact that wavelet processing allows taking into account the particular characteristics of the images under study by decomposing source data into a plurality of approximate and detail coefficients, in particular by image edge detection [8]. Wavelet analysis is based on wavelet transform. The wavelet transform is a signal decomposition (e.g. of some image) by the system of wavelets. Wavelets are obtained by shifting and scaling a single function-parent wavelet [9].

The general ideology of wavelet analysis for images of cytological preparations is as follows [10] [11]: 
1. The horizontal discontinuities of the original image are analyzed.

2. The vertical discontinuities of the original image are analyzed.

This analysis is carried out using wavelets. To do this, there's the use of two continuous and integral along the whole axis functions [9] [12]:

-wavelet-function $\phi(t)$ with zero integral value

$$
\int_{-\infty}^{\infty} \phi(t) \mathrm{d} t=0
$$

determining the details of the signal and generating extended fractions;

-scaling function $\varphi(t)$ with a unit value of integral

$$
\int_{-\infty}^{\infty} \varphi(t) \mathrm{d} t=1
$$

determining a rough approximation of signal and generating approximation coefficients.

3. The matrix of the processed image is formed. This is done using a matrix of horizontal and matrix vertical discontinuities of the original image (see for more details [8] [11] [13] [14]).

To ensure the reliability of the detection of discontinuities, it is necessary to carry out the procedure of enhancing the contrast of the image. This is one of the conditions for improving the quality of image processing of cytological preparations [15].

\subsection{Color Systems for Image Analysis}

Typically, when processing images, the RGB color model is considered [16]. The image in this color model consists of three color channels-R (red), G (green) and $B$ (blue) [17]. Then each new color in the image is the fusion of the primary colors: $\mathrm{R}, \mathrm{G}$ and $\mathrm{B}$ :

$$
C Q(i, j)=R(i, j) \cap G(i, j) \cap B(i, j)
$$

where

$C Q(i, j)$-definition of colors in the original image $Q$ at the point $(i, j)$ in the model RGB,

$R(i, j)$-definition of colors in the channel $R$ at the point $(i, j)$ for the original image $Q$,

$G(i, j)$-definition of colors in the channel $G$ at the point $(i, j)$ for the original image $Q$,

$B(i, j)$-definition of colors in the channel $B$ at the point $(i, j)$ for the original image $Q$,

$\cap$ - color fusion operation in the model RGB.

If we apply the procedure of changing the image contrast to each channel, then we can get an error. This is due to the fact that we will violate the initial conditions for the formation of a color image. Therefore, to change the contrast of the image, we must:

-make the conversion from a color image to a black and white image; 
-do change the contrast for the black-and-white image.

In this case, we will also have errors. This is due to the transition from a color image to a black and white image.

But we can move from the color RGB model to the HSV (H-hue, S-saturation, $\mathrm{V}$-volume or brightness) model. Thus, we have other components for color formation on the original image:

$$
Z Q(i, j)=H(i, j) \cap S(i, j) \cap V(i, j)
$$

where

$Z Q(i, j)$-definition of colors in the original image $Q$ at the point $(i, j)$ in the model HSV,

$H(i, j)$-component of color in the channel $H$ at the point $(i, j)$ for the original image $Q$,

$S(i, j)$-component of color in the channel $S$ at the point $(i, j)$ for the original image $Q$,

$V(i, j)$-component of color in the channel $V$ at the point $(i, j)$ for the original image $Q$,

$\cap$-color fusion operation in the model HSV.

Model HSV is a non-linear transformation of the RGB model. The relationship between RGB and HSV is implemented as follows [18]:

$$
H=\left\{\begin{array}{l}
0, \text { if } M A X=M I N, \\
60 \times \frac{G-B}{M A X-M I N}, \text { if } M A X=R, G \geq B, \\
60 \times \frac{G-B}{M A X-M I N}+360, \text { if } M A X=R, G<B, \\
60 \times \frac{B-R}{M A X-M I N}+120, \text { if } M A X=G, \\
60 \times \frac{R-G}{M A X-M I N}+240, \text { if } M A X=B, \\
S=\left\{\begin{array}{l}
0, \text { if } M A X=0, \\
1-\frac{M I N}{M A X}, \text { if } M A X>0, \\
V=M A X,
\end{array}\right.
\end{array}\right.
$$

where

$$
\begin{aligned}
M A X & =\max (R, G, B), \\
M I N & =\min (R, G, B),
\end{aligned}
$$

$R, G, B$-the corresponding channel values in the model RGB,

$H, S, V$-the corresponding channel values in the model HSV.

Then, to change the contrast, it is necessary to carry out the procedure for channel V in the model HSV.

\subsection{Data for Analysis}

In order to identify the possibility of using wavelet analysis as a processing tool 
for cytology preparations images for various color models, some image have been selected. The test image is shown in Figure 1 (image cells in pernicious anemia). This image is analyzed in our other articles and gives an opportunity to make a comparative analysis [8].

This image reflects common features in the analysis of cytological drugs. We can see the different types of cells, the complex structure of the cell. This image allows us to test the possibilities of wavelet analysis, which we described earlier [8] [10] [11].

In Figure 1, we see one of the varieties of pernicious anemia-this is megaloblastic anemia. Megaloblastic anemia is a blood disorder marked by the appearance of a very large red cells (cells of blue color) that crowd out of a healthy cells (cells of red-pink color), causing anemia.

\section{Results}

We set the task of comparing the results of applying wavelet analysis for different input images. To do this, we consider the results of wavelet analysis:

-for the original image (see Figure 1):

-for the image for which we applied the procedure for changing the contrast in the color RGB model;

-for the image for which we applied the procedure for changing the contrast in the color HSV model.

Figure 2 shows the original image, for which we applied the procedure for changing the contrast in the color RGB model. To change the contrast, we use the equalization procedure (aligning the histogram of the brightness values of the image elements [19]). In Figure 2 the image is in grayscale.

Figure 3 shows the original image, for which we applied the procedure for changing the contrast in the color HSV model. To change the contrast, we also use the equalization procedure. In Figure 3 the image is in grayscale (gray-level).

We can observe significant differences between the images, which are shown in Figure 2 and Figure 3.

Figure 4 shows the original image in grayscale (gray-level).

To the image in Figure 4, in Figure 2 and the image in Figure 3 we applied wavelet analysis. In Figures 5-7 shows the results of wavelet analysis.

The best result of wavelet analysis is shown in Figure 5 and Figure 6. These

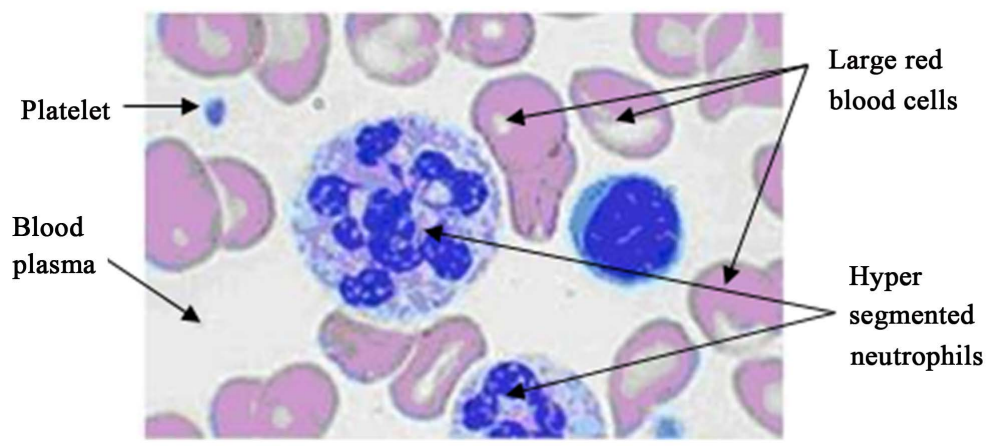

Figure 1. Image cells in pernicious anemia. 


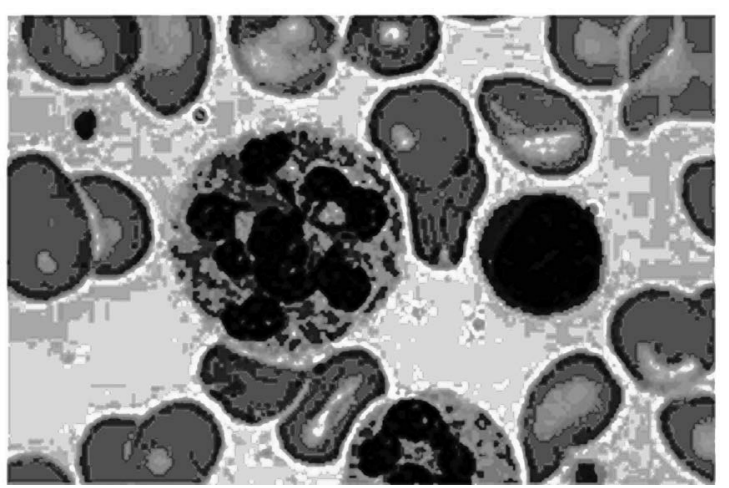

Figure 2. Change the contrast in the RGB model.

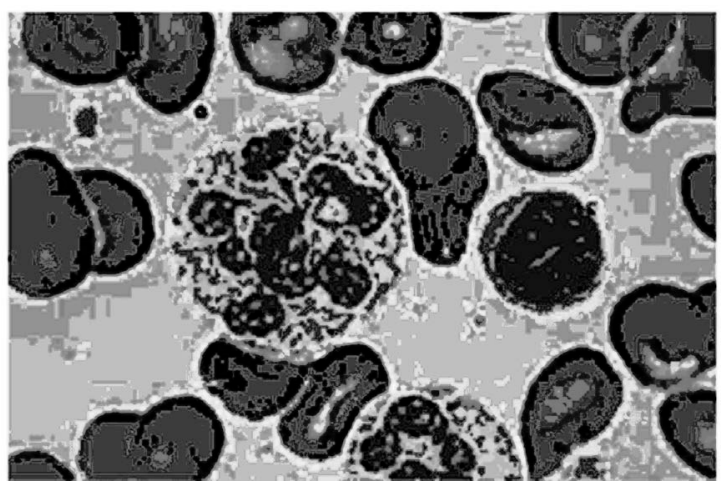

Figure 3. Change the contrast in the HSV model.

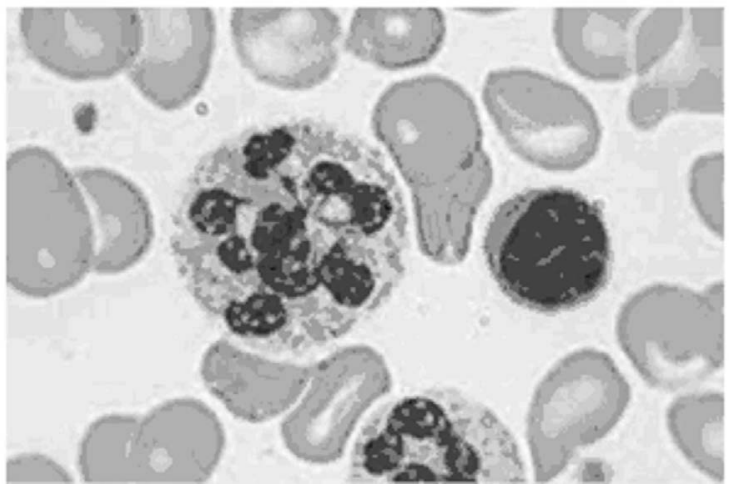

Figure 4. The original image in grayscale.

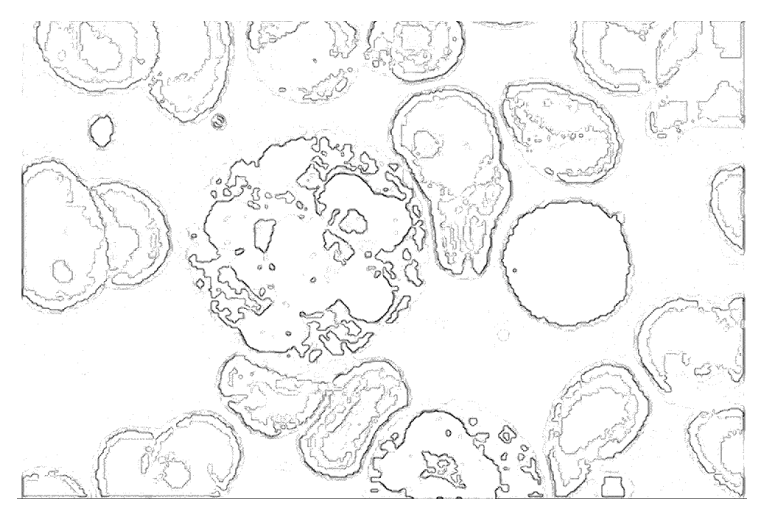

Figure 5. Wavelet analysis (for Figure 2). 


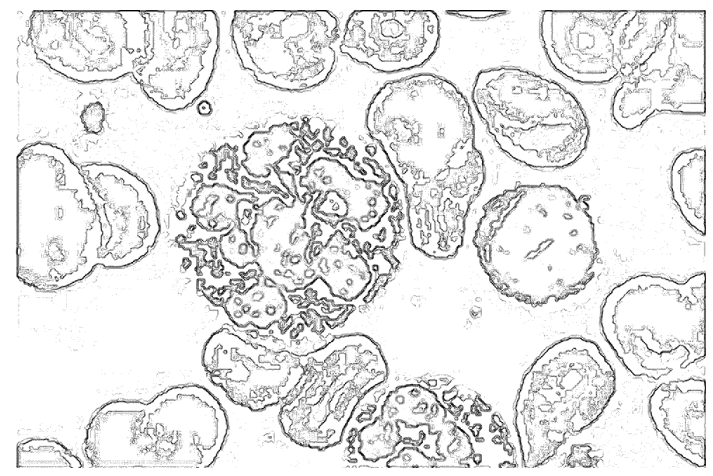

Figure 6. Wavelet analysis (for Figure 3).

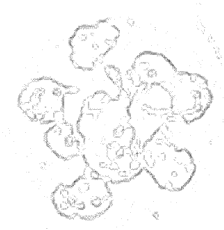

Figure 7. Wavelet analysis (for Figure 4).

are the images for which the procedure for changing the contrast was made (see Figure 2 and Figure 3). We also see that in Fig. 6, more details are shown than in Figure 5. In Figure 6 we clearly see the internal structure of the cells. Thus, changing the contrast in the HSV model gives the best result for wavelet analysis.

Therefore, we can say that the general procedure for wavelet analysis of cytological preparations in different color systems is as follows:

-obtaining a color image of cytological preparation;

-converting the image into the HSV color model;

-change the image contrast in the HSV model;

-application of the wavelet analysis procedure and obtaining of the final results.

\section{Conclusion}

The article discusses the possibility of using the wavelet analysis in various color models. We compare the use of wavelet analysis in the color model RGB and HSV. To do this, we use the procedure of changing the original image contrast in the color models RGB and HSV. We showed that changing the contrast in the HSV model gives the best result for wavelet analysis. The advantages of this result are the obtaining of a large number of additional details for the original image. This allows qualitatively allocating: cells' edges, cell nuclei, revealing in more detail textural features of cells' images, which allows analyzing cell structure. 
This allows you to build a better system for analysis of cytological specimen's images.

\section{References}

[1] Hameed, B.M., Aboob, H.S. and Musa, R.J. (2016) Immunohistochemical Expression of VEGF in Relation to VEGFR and CD34 in NHL Using Digital Image Analysis System. Iraqi Journal of Hematology, 5, 19-31.

[2] Newton, T. M. and Reid, E. (2016) An Automated Image Analysis System to Quantify Endosomal Tubulation. PloS One, 11, e0168294.

https://doi.org/10.1371/journal.pone.0168294

[3] Myburgh, H.C., van Zijl, W.H., Swanepoel, D., Hellström, S. and Laurent, C. (2016) Otitis Media Diagnosis for Developing Countries Using Tympanic Membrane ImageAnalysis. EBioMedicine, 5, 156-160. https://doi.org/10.1016/j.ebiom.2016.02.017

[4] Singh, S. and Gupta, R. (2012) Identification of Components of Fibroadenoma in Cytology Preparations Using Texture Analysis: A Morphometric Study. Cytopathology, 23, 187-191. https://doi.org/10.1111/j.1365-2303.2011.00854.x

[5] Mahendran, G., Babu, R. and Sivakumar, D. (2014) Automatic Segmentation and Classification of Pap Smear Cells. International Journal of Management, IT and Engineering, 4, 100-108.

[6] Saha, M., Agarwal, S., Arun, I., Ahmed, R., Chatterjee, S., Mitra, P. and Chakraborty, C. (2015) Histogram Based Thresholding for Automated Nucleus Segmentation Using Breast Imprint Cytology. In: Advancements of Medical Electronics, Springer India, 49-57. https://doi.org/10.1007/978-81-322-2256-9_5

[7] George, Y.M., Bagoury, B.M., Zayed, H.H. and Roushdy, M.I. (2013) Automated Cell Nuclei Segmentation for Breast Fine Needle Aspiration Cytology. Signal Processing, 93, 2804-2816. https://doi.org/10.1016/j.sigpro.2012.07.034

[8] Lyashenko, V.V., Babker, A.M.A.A. and Kobylin, O.A. (2016) The Methodology of Wavelet Analysis as a Tool for Cytology Preparations Image Processing. Cukurova Medical Journal, 41, 453-463. https://doi.org/10.17826/cukmedj.237468

[9] Kingsbury, N. (1999) Image Processing with Complex Wavelets. Philosophical Transactions of the Royal Society of London A: Mathematical, Physical and Engineering Sciences, 357, 2543-2560. https://doi.org/10.1098/rsta.1999.0447

[10] Kobylin, O. and Lyashenko, V. (2014) Comparison of Standard Image Edge Detection Techniques and of Method Based on Wavelet Transform. International Journal of Advanced Research, 2, 572-580.

[11] Lyashenko, V., Matarneh, R., Kobylin, O. and Putyatin, Y. (2016) Contour Detection and Allocation for Cytological Images Using Wavelet Analysis Methodology. International Journal of Advance Research in Computer Science and Management Studies, 4, 85-94.

[12] Heil, C.E. and Walnut, D.F. (1989) Continuous and Discrete Wavelet Transforms. SIAM Review, 31, 628-666. https://doi.org/10.1137/1031129

[13] Lyashenko, V.V., Babker, A.M.A.A. and Kobylin, O.A. (2016) Using the Methodology of Wavelet Analysis for Processing Images of Cytology Preparations. National Journal of Medical Research, 6, 98-102.

[14] Lyashenko, V., Matarneh, R. and Kobylin, O. (2016) Contrast Modification as a Tool to Study the Structure of Blood Components. Journal of Environmental Science, Computer Science and Engineering \& Technology, 5, 150-160.

[15] Dey, N., Ashour, A.S., Ashour, A.S. and Singh, A. (2015) Digital Analysis of Micro- 
scopic Images in Medicine. Journal of Advanced Microscopy Research, 10, 1-13. https://doi.org/10.1166/jamr.2015.1229

[16] Cheng, H.D., Jiang, X.H., Sun, Y. and Wang, J. (2001) Color Image Segmentation: Advances and Prospects. Pattern Recognition, 34, 2259-2281.

https://doi.org/10.1016/S0031-3203(00)00149-7

[17] Comaniciu, D. and Meer, P. (1997) Robust Analysis of Feature Spaces: Color Image Segmentation. Proceedings of IEEE Computer Society Conference on Computer Vision and Pattern Recognition, Santa Barbara, 17-19 June 1997, 750-755. https://doi.org/10.1109/cvpr.1997.609410

[18] Chen, W., Shi, Y.Q. and Xuan, G. (2007) Identifying Computer Graphics Using HSV Color Model and Statistical Moments of Characteristic Functions. IEEE International Conference on Multimedia and Expo, Beijing, 2-5 July 2007, 1123-1126. https://doi.org/10.1109/icme.2007.4284852

[19] Semmlow, J.L. and Griffel, B. (2014) Biosignal and Medical Image Processing. CRC Press, Boca Raton.

Submit or recommend next manuscript to OALib Journal and we will provide best service for you:

- Publication frequency: Monthly

- 9 subject areas of science, technology and medicine

- Fair and rigorous peer-review system

- Fast publication process

- Article promotion in various social networking sites (LinkedIn, Facebook, Twitter, etc.)

- Maximum dissemination of your research work

Submit Your Paper Online: Click Here to Submit

Or Contact service@oalib.com 